\title{
A Statistical Inverse Method for Gridding Passive Microwave Data With Mixed Measurements
}

\author{
Rafael Grimson ${ }^{\circledR}$, Juan Lucas Bali ${ }^{\circledR}$, Mariela Rajngewerc, Laura San Martín, and \\ Mercedes Salvia, Member, IEEE
}

\begin{abstract}
When a passive microwave footprint intersects objects on the ground with different spectral characteristics, the corresponding observation is mixed. The retrieval of geophysical parameters is limited by this mixture. We propose to partition the study region into objects following an object-based image analysis procedure and then to refine this partition into small cells. Then, we introduce a statistical method to estimate the brightness temperature (TB) of each cell. The method assumes that TB of the cells corresponding to the same object is identically distributed and that the TB heterogeneity within each cell can be neglected. The implementation is based on an iterative expectation-maximization algorithm. We evaluated the proposed method using synthetic images and applied it to grid the TBs of sample AMSR -2 real data over a coastal region in Argentina.
\end{abstract}

Index Terms-Expectation-maximization (EM) algorithms, inverse problems, passive microwave remote sensing.

\section{INTRODUCTION}

$\mathbf{M}$ ICROWAVE radiometers typically measure the (horizontally or vertically) polarized microwave radiation emitted at a given frequency from the earth's surface within their field of view. These measurements are expressed as brightness temperatures (TBs). The sensitivity of microwave measurements to soil and vegetation properties was proven by several theoretical and experimental investigations [1], [2]. At lower frequencies, passive microwave observations are highly sensitive to the surface and subsurface properties such as soil moisture and soil texture. Because of this sensitivity, land emissivity estimates are appropriate for the retrieval of geophysical parameters such as soil moisture estimation [3], freeze/thaw state [4], land surface temperature [5], flooded area estimation [6], and vegetation structure [7].

Manuscript received July 4, 2018; revised August 6, 2018; accepted August 15, 2018. This work was supported by Agencia Nacional de Promoción Científica y Tecnológica of Argentina through FONCyT MinCyT under Grant PICT-2014-0824 and Grant PICT-2016-4089. (Corresponding author: Rafael Grimson.)

R. Grimson and L. S. Martin are with the Instituto de Investigacion e Ingenieria Ambiental, Universidad Nacional de San Martín, San Martín 1650, Argentina, and also with the Consejo Nacional de Investigaciones Cientificas y Tecnologicas, CABA 1425, Argentina (e-mail: rgrimson@ unsam.edu.ar).

J. L. Bali is with the Instituto de Investigaciones Científicas y Tecnicas para la Defensa, Villa Martelli 1603, Argentina, and also with the Consejo Nacional de Investigaciones Cientificas y Tecnologicas, CABA 1425, Argentina.

M. Rajngewerc is with the Instituto de Investigacion e Ingenieria Ambiental, Universidad Nacional de San Martín, San Martín 1650, Argentina.

M. Salvia is with the Instituto de Astronomia y Fisica del Espacio, Universidad de Buenos Aires, Buenos Aires 1053, Argentina, and also with the Consejo Nacional de Investigaciones Cientificas y Tecnologicas, CABA 1425, Argentina.

Color versions of one or more of the figures in this paper are available online at http://ieeexplore.ieee.org.

Digital Object Identifier 10.1109/TGRS.2018.2866196
Spaceborne radiometer systems supply frequent coverage of the globe. This coverage is provided in all weather conditions and during day and night since the atmosphere is effectively transparent for most microwaves frequencies and measurements are independent of solar illumination. Also, long time series of observations are available since microwave observations over the globe by different satellite radiometers started in the 1970s [1], [2].

However, in order to detect the low radiation emitted at a given frequency, the field of view of passive sensors must be large enough to integrate sufficient energy. This results in a low spatial resolution in the order of tens of kilometers for satellite observations.

Typically, microwave radiometer antennas show a conical scanning scheme, where the $3-\mathrm{dB}$ contour of the antenna gain function projects an ellipse on the Earth's surface with its minor axis along the scan direction. The size of these ellipses depends on the sensed frequency and causes a substantial overlapping of the measurements.

If a footprint intersects objects on the ground with different spectral characteristics, the corresponding antenna TB will be contaminated. For instance, an observation whose footprint intersects a coastline would measure a weighted average of land and sea TBs. The retrieval of geophysical parameters from these measurements is limited by the contamination. In fact, AMSR-2 soil moisture product shows erroneously high values near coastlines and large lakes, while losses in the estimation of sea surface temperature caused by land effects extend as far as $100 \mathrm{~km}$ from coastlines [8]. In many cases, the retrieval of geophysical parameters relies on the use of indices based on the combination of TBs sensed at different frequencies, such as the frequency index [9]

$$
\mathrm{FI}=\frac{2\left(\mathrm{~TB}_{v}^{\mathrm{K}_{\mathrm{a}}}-\mathrm{TB}_{v}^{X}\right)}{\mathrm{TB}_{v}^{\mathrm{K}_{\mathrm{a}}}+\mathrm{TB}_{v}^{X}}
$$

which involves vertically polarized emissions in the $\mathrm{K}_{\mathrm{a}}$ - and $\mathrm{X}$-bands. These indices should not be computed directly from footprints of different size, since they show different amounts of contamination. Thus, a technique capable of separating signals from mixed measurements would be of significant value for the retrieval of physical parameters in coastal and border areas.

The main approach taken to address image interpolation and resolution matching consists in the application of Backus-Gilbert (BG) optimal interpolation theory [10], as formulated for this class of problems by Stogryn [11] and first applied to SSM/I data by Poe [12]. Farrar et al. [13] 
used this approach to match the observations acquired at different frequencies in a multi-frequency analysis and it is still used by the Japan Aerospace Exploration Agency to compute AMSR-2 L1R products [8]. BG solution is a compromise between resolution and noise. The resolution is typically quantified using an isotropic penalty function that does not take into account the different spectral objects in the scene. Thus, the resulting method does not allow the separation of information from mixed measurements. Consequently, when BG is directly applied to grid passive microwave data with mixed measurements, a smoothing effect can be perceived around the borders of the objects.

There are at least two examples in the literature where the study region is partitioned into objects following a ground-based criterion, and then refined into cells with homogeneous TBs. Bellerby et al. [14] presented a technique to separate uncontaminated land and sea TBs from mixed coastal measurements, combining a model of the antenna TB with a digitalized cartographic representation of the coastline. For a small coastal region, they assume that the sea and land temperature fields are uniform, and consider the linear system with two unknowns ( $T_{\text {Land }}$ and $T_{\text {Sea }}$ ) resulting from nine $(3 \times 3)$ measurements in this region. Then, they use least-squares optimization to obtain the estimates for the actual land and sea TB present in the small region.

Limaye et al. [15] proposed a technique to estimate microwave TBs of agricultural fields from airborne measurements taken by the PALS sensor. To apply this technique, the study region is segmented into spatially contiguous objects sharing spectral characteristics (agricultural fields) by an image analyst using optical images. The TB is assumed to be constant over each segment. This, together with the antenna model, leads to an overdetermined linear system that is solved by minimizing the sum of residuals. The successful application of the deconvolution technique in that study can be attributed to the relatively large size of the segments with respect to the sampling resolution and the consequent observation over-sampling, together with a precise groundbased tiling of the study region into segments with almost constant TB.

In this article, we propose to partition the study region into objects following an object-based image analysis procedure [16], [17] and refine this partition into cells of TB. Assuming similar size (Section II-B). Then, assuming a constant TB over each cell, we introduce the discrete antenna model. In Section III, we present the statistical inverse method used to transform the antennas TB into cells TB. This method assumes that the TB corresponding to cells of the same type (i.e., that belong to the same object) can be modeled by the same normal distribution. The most likely parameters of these distributions are found with an expectation-maximization (EM) algorithm and then used to estimate the TB of the cells. In Section IV-A, we use synthetic images to evaluate the proposed methods by means of Monte Carlo experiments. Then, we apply the methods to a real case (Section IV-E). Finally, in Section V, we discuss the potential and limitations of the proposed methods.

\section{DiscRETE MODEL}

\section{A. Discretization of Antenna Brightness Temperature Model}

Assuming that the microwave emission upwelling from the study area, $\operatorname{TB}(\rho)$, is independent of the measurement direction (over the angular region of interest) and that its time variation (during the measurements) may be neglected, the following equation [11], [12], [18] is valid for a single measured antenna TB, $T_{A}$ :

$$
T_{A}=\int_{E} G(\rho) \mathrm{TB}(\rho) d A+\varepsilon .
$$

Here, the variable $\varepsilon$ represents the observational error and the function $G$ represents the projection of the antenna gain function onto the Earth surface. $G$ may be considered known since it depends only on the position and characteristics of the sensor (altitude, incidence angle, and shape of the sensor antenna response function).

It is evident that the problem of inferring TB from a finite set of measurements is an underconstrained problem. Usually, the region is gridded and each cell of the grid is assumed to have a uniform TB. Assuming constant TB for each cell, (2) is transformed into a linear equation relating the cells TB with the measured antenna TB. If the study region is partitioned into $n$ cells, $C_{1}, \ldots, C_{n}$, each of them with uniform TB $x_{1}, \ldots, x_{n}$, and we have a set of $m$ measurements $y_{1}, \ldots, y_{m}$, then the corresponding linear system can be expressed as

$$
\boldsymbol{y}=\mathbf{K} \boldsymbol{x}+\boldsymbol{\varepsilon}
$$

where $\boldsymbol{y}=\left(y_{1}, \ldots, y_{m}\right)$ represents the $m$ measured antenna temperatures, $\boldsymbol{x}=\left(x_{1}, \ldots, x_{n}\right)$ represents the cells temperatures, $K \in \mathbb{R}^{n \times m}$ is a kernel or weight matrix where $K_{i j}$ is the $j$ th cell fractional contribution to the $i$ th measurement, $K_{i j}=\int_{C_{j}} G_{i}(\rho) d A$, and $\boldsymbol{\varepsilon}$ is the vector of observational errors, which are assumed to be independent and identically distributed normal random variables, $\varepsilon_{i} \sim \mathcal{N}\left(0, \sigma_{\text {obs }}^{2}\right)$. In this model, the variables $y_{1}, \ldots, y_{m}$ are observable variables, whereas the variables $x_{1}, \ldots, x_{n}$ are state variables.

The problem of estimating $\boldsymbol{x}$ from the observations is an inverse problem. In general, a method to solve this inverse problem must augment the information given by the observations with some a priori knowledge of the nature of the solution.

\section{B. Construction of the Retrieval Grid}

We assume that the spatial boundaries of the contributing emitting sources are known a priori and use them to construct a retrieval grid. We propose to partition the study region into objects having homogeneous spectral characteristics, as follows from an object-based image analysis of the study region. We emphasize that we do not assume that these objects have constant TB, but that the TB field within each object can be modeled by a common probabilistic distribution.

These objects may be different surface cover types (such as land and sea), agricultural fields of a given crop, or any partition of the scene into homogeneous ecogeomorphologic objects. We refer to this partition of the study region as the ground-based partition and label the objects in this partition, 


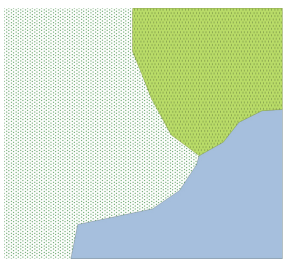

(a)

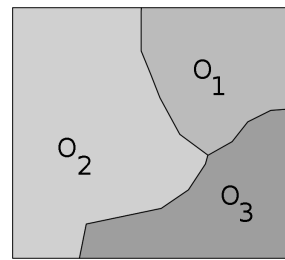

(b)

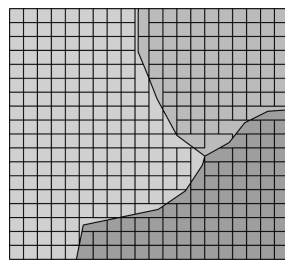

(c)
Fig. 1. Representation of (a) generic study region including three spectrally different landscapes, (b) its ground-based partition into spectral objects, and (c) refinement of this partition into a retrieval grid composed of cells of three different types.

$O_{1}, \ldots, O_{k}$, for future reference. In Fig. 1(b), a partition into three objects is shown.

Since the resulting objects may be of different sizes, and, in particular, too big, we propose to partition these objects into cells of a similar given size. Each cell is labeled, $l_{1}, \ldots, l_{n}$, with $l_{i} \in\left\{O_{1}, \ldots, O_{k}\right\}$, to identify its object of origin. We refer to this refinement of the ground-based partition as the retrieval grid.

Under the assumption that the TB field is constant over each cell, we obtain a linear system (as (3)) expressing the measured TBs as linear combinations of the contributing-cells TBs.

\section{Resolution Methods}

Different methods may be used to solve the inverse problem over a resolution grid. We first introduce two benchmark methods that will be compared with the proposed technique. These two methods do not consider the type of each cell. Then, we introduce the proposed statistical method. Finally, we discuss some computational aspects of the algorithms.

\section{A. Least-Squares Method}

If the resolution grid is coarse, the resulting linear system is overdetermined and typically has no solution. In this case, the system may be solved using least-squares optimization

$$
\hat{\boldsymbol{x}}=\underset{\boldsymbol{x}}{\operatorname{argmin}}\|\mathbf{K} \boldsymbol{x}-\boldsymbol{y}\|^{2} .
$$

If the discretization is fine, the system is underdetermined and has typically infinitely many solutions. The choice of a solution must include some a priori information.

\section{B. Backus-Gilbert Method}

The second benchmark method is the BG method [10]. Consider the problem of estimating the TB $x_{C}$ for a fixed cell $C$ in the retrieval grid. In our context, the BG approach proposes to approximate $x_{C}$ as an affine combination of the measurements $y_{i}$

$$
x_{C}=\sum_{i=1}^{m} a_{i} y_{i}, \quad \sum_{i=1}^{m} a_{i}=1
$$

where the coefficients $a_{i}$ are to be determined. Ideally, the kernel resulting from the corresponding combination of the antenna gain functions should be proportional to the characteristic function of the cell $\sum_{i=1}^{m} a_{i} G_{i} \sim \chi_{C}$. However, with a finite number of measurements, it is not possible to choose the coefficients to produce this kernel, so an approximation that closely produces the ideal behavior is sought. In our case, we measure the resolution error for a tuple $\boldsymbol{a}=\left(a_{1}, \ldots, a_{m}\right)$ of coefficients as the contribution of the points out of the cell $C$ to the kernel, weighted by the distance to the cell $C$

$$
Q_{0}(\boldsymbol{a})=\int\left(\sum_{i=1}^{m} a_{i} G_{i}(\rho)\right)^{2} \operatorname{dist}^{2}(\rho, C) d A
$$

where $\operatorname{dist}(\rho, C)$ is the distance from $\rho$ to the cell $C$. Other objective functions were tested, but this resulted in the best approximation performance, as measured by the root-meansquared error (RMSE) (cf. [11]).

Also, in order to measure the propagation of the experimental errors, BG proposal considers the term

$$
e^{2}=a S_{\varepsilon} a^{T}
$$

where $S_{\varepsilon}$ is the error covariance matrix of the measurements. In our case, since we assume that the measurement errors are independent and identically distributed, $\boldsymbol{S}_{\boldsymbol{\varepsilon}}$ is a scalar matrix.

The BG method seeks to minimize an objective function that results from a compromise between the resolution term (6) and the error term (7). It is given by

$$
Q=Q_{0} \cos (\gamma)+e^{2} \omega \sin (\gamma)
$$

where $\omega$ is simply a scale parameter and $\gamma$ is a fine-tuning parameter which, when varied from 0 to $(\pi / 2)$, places less emphasis on the resolution and more on reducing the noise in the estimate of $x_{C}=\mathrm{TB}(C)$. The parameters were chosen to minimize the RMSE in our synthetic scenarios.

The solution to the minimization problem $\operatorname{argmin}_{\boldsymbol{a}} Q(\boldsymbol{a})$ constrained to $\sum_{i=1}^{m} a_{i}=1$ is simple. We refer the reader to [11] for its derivation and closed solution formula.

\section{Statistical Approach}

In this approach, $\boldsymbol{x}, \boldsymbol{y}$, and $\boldsymbol{\varepsilon}$ are considered random variables. We propose to model the temperature of each cell with a univariate normal distribution whose parameters depend only on the type of the cell, $x_{i} \sim \mathcal{N}\left(\mu_{l_{i}}, \sigma_{l_{i}}^{2}\right)$ for $i=1, \ldots, n$.

Let $\boldsymbol{\theta}=\left(\mu_{1}, \ldots, \mu_{k}, \sigma_{1}^{2}, \ldots, \sigma_{k}^{2}\right)$ comprise the parameters of our a priori constraint to the problem, i.e., the mean and variance of the TBs for each one of the different $k$ cell types. Hence, for a given $\boldsymbol{\theta}$, the cell temperatures $\boldsymbol{x}$ are modeled using a multivariate normal distribution with mean vector $\boldsymbol{\mu}_{\boldsymbol{x}}=\left(\mu_{l_{1}}, \ldots, \mu_{l_{n}}\right)$ and a diagonal covariance matrix $\boldsymbol{S}_{\boldsymbol{x}}$, with $\operatorname{diag}\left(\boldsymbol{S}_{\boldsymbol{x}}\right)=\left(\sigma_{l_{1}}^{2}, \ldots, \sigma_{l_{n}}^{2}\right)$.

The errors $\varepsilon_{1}, \ldots, \varepsilon_{m}$ are also modeled as independent and identically distributed, $\varepsilon_{i} \sim \mathcal{N}\left(0, \sigma_{\text {obs }}^{2}\right)$. Here, $\sigma_{\text {obs }}^{2}$ represents the observational error variance and is assumed to be known (though this assumption may be relaxed and a cross-validation procedure can be used to determine this value).

Using Bayes theorem, we have

$$
p(\boldsymbol{x} \mid \boldsymbol{y}, \boldsymbol{\theta})=\frac{p(\boldsymbol{y} \mid \boldsymbol{x}) \cdot p(\boldsymbol{x} \mid \boldsymbol{\theta})}{p(\boldsymbol{y} \mid \boldsymbol{\theta})} \propto p(\boldsymbol{y} \mid \boldsymbol{x}) \cdot p(\boldsymbol{x} \mid \boldsymbol{\theta}) .
$$


The expression $p(\boldsymbol{y} \mid \boldsymbol{x})$ represents the relative likelihood of having $\boldsymbol{\varepsilon}=(\boldsymbol{y}-\boldsymbol{K} \boldsymbol{x})$ as measurement errors. The variable $\boldsymbol{\varepsilon}$ has a multivariate normal distribution with zero mean and a scalar covariance matrix, $S_{\varepsilon}$, with the variance of the observational errors, $\sigma_{\mathrm{obs}}^{2}$, as entries in the diagonal. On the other hand, $p(\boldsymbol{x} \mid \boldsymbol{\theta})$ represents the relative likelihood of the temperature values $\boldsymbol{x}$ for the cells in the image, as stated by the prior with parameters $\boldsymbol{\theta}$.

If $\boldsymbol{\theta}$ is a known parameter, it is not hard to obtain the posterior distribution $p(\boldsymbol{x} \mid \boldsymbol{y}, \boldsymbol{\theta})$ for the cell TB, which will be a multivariate normal [19] with mean

$$
\hat{\boldsymbol{x}}=\left(\boldsymbol{S}_{\boldsymbol{x}}{ }^{-1}+\boldsymbol{K}^{t} \boldsymbol{S}_{\boldsymbol{\varepsilon}}^{-1} \boldsymbol{K}\right)^{-1}\left(\boldsymbol{S}_{\boldsymbol{x}}{ }^{-1} \boldsymbol{\mu}_{\boldsymbol{x}}+\boldsymbol{K}^{t} \boldsymbol{S}_{\boldsymbol{\varepsilon}}{ }^{-1} \boldsymbol{y}\right)
$$

and covariance matrix

$$
\hat{\boldsymbol{S}}=\left(\boldsymbol{S}_{\boldsymbol{x}}{ }^{-1}+\boldsymbol{K}^{t} \boldsymbol{S}_{\boldsymbol{\varepsilon}}^{-1} \boldsymbol{K}\right)^{-1} .
$$

To emphasize the origin of the parameters involved in the computation of $\hat{\boldsymbol{S}}$ and $\hat{\boldsymbol{x}}$, we may write $\hat{\boldsymbol{S}}_{\boldsymbol{\theta}}$ and $\hat{\boldsymbol{x}}_{\boldsymbol{\theta}}$, respectively.

Since $\boldsymbol{\theta}$ is typically unknown, we propose to use its maximum likelihood estimate from the measurements $\boldsymbol{y}$. This estimate, $\hat{\boldsymbol{\theta}}$, is computed using an EM procedure [20]. Here, $\boldsymbol{x}$ is a latent variable. Consider the likelihood function $L(\boldsymbol{\theta})=p(\boldsymbol{y} \mid \boldsymbol{\theta})$. Marginalizing the joint distribution $p(\boldsymbol{y}, \boldsymbol{x} \mid \boldsymbol{\theta})$, we obtain

$$
L(\boldsymbol{\theta})=p(\boldsymbol{y} \mid \boldsymbol{\theta})=\int p(\boldsymbol{y}, \boldsymbol{x} \mid \boldsymbol{\theta}) d \boldsymbol{x} .
$$

In order to maximize this usually intractable quantity, the EM procedure proposes an iterative mechanism as follows [21]:

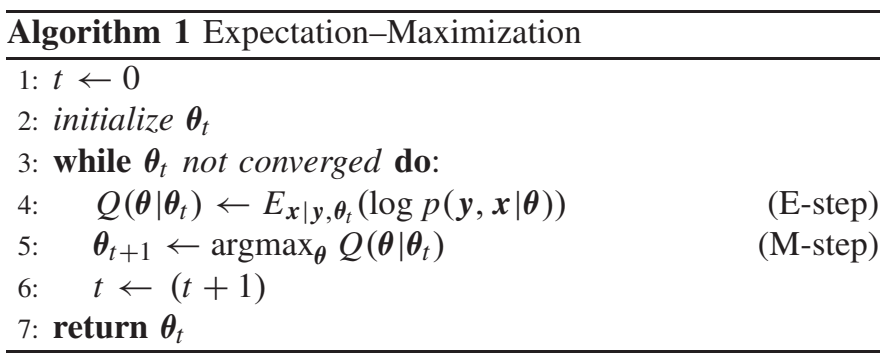

For the E-step, we need $\mathrm{n}$ exapression for $p\left(\boldsymbol{x} \mid \boldsymbol{y}, \boldsymbol{\theta}_{t}\right)$. This posterior probability has a multivariate normal distribution with mean $\hat{\boldsymbol{x}}_{\boldsymbol{\theta}_{t}}$ and covariance matrix $\hat{\boldsymbol{S}}_{\boldsymbol{\theta}_{t}}$ given by (10) and (11).

In the M-step, we have to maximize the expectation of $\log p(\boldsymbol{y}, \boldsymbol{x} \mid \boldsymbol{\theta})=\log p(\boldsymbol{y} \mid \boldsymbol{x})+\log p(\boldsymbol{x} \mid \boldsymbol{\theta})$. Since the first term does not depend on $\boldsymbol{\theta}$, we can omit it for the maximization. Hence, we have to maximize $E\left(-(1 / 2)\left(x-\mu_{x}\right)^{t} S_{x}{ }^{-1}(x-\right.$ $\left.\left.\boldsymbol{\mu}_{\boldsymbol{x}}\right)-\log \left(\left|\boldsymbol{S}_{\boldsymbol{x}}\right|\right)\right)$ where $\boldsymbol{x}$ has a multivariate normal distribution with mean $\hat{\boldsymbol{x}}_{\boldsymbol{\theta}_{t}}$ and covariance $\hat{\boldsymbol{S}}_{\boldsymbol{\theta}_{t}}$. Completing the square, it is possible to rewrite this expectation so as to obtain

$$
-\log \left(\left|\boldsymbol{S}_{\boldsymbol{x}}\right|\right)-\operatorname{tr}\left(\boldsymbol{S}_{\boldsymbol{x}}{ }^{-1} \hat{\boldsymbol{S}}_{\boldsymbol{\theta}_{t}}\right)-\left(\hat{\boldsymbol{x}}_{\boldsymbol{\theta}_{t}}-\boldsymbol{\mu}_{\boldsymbol{x}}\right)^{t} \boldsymbol{S}_{\boldsymbol{x}}{ }^{-1}\left(\hat{\boldsymbol{x}}_{\boldsymbol{\theta}_{t}}-\boldsymbol{\mu}_{\boldsymbol{x}}\right) .
$$

In order to maximize this expression, we differentiate it with respect to each component of $\boldsymbol{\theta}=\left(\mu_{1}, \ldots, \mu_{k}, \sigma_{1}^{2}, \ldots, \sigma_{k}^{2}\right)$. We obtain that the maximum is achieved when

$$
\mu_{j}=\frac{1}{n_{j}} \sum_{i=1}^{n_{j}} P_{j}\left(\hat{\boldsymbol{x}}_{\boldsymbol{\theta}_{t}}\right)
$$

and

$$
\sigma_{j}^{2}=\frac{1}{n_{j}}\left(\left\|P_{j}\left(\operatorname{diag}\left(\hat{\boldsymbol{S}}_{\boldsymbol{\theta}_{t}}\right)\right)\right\|_{1}+\left\|P_{j}\left(\hat{\boldsymbol{x}}_{\boldsymbol{\theta}_{t}}-\boldsymbol{\mu}_{\boldsymbol{x}}\right)\right\|_{2}^{2}\right)
$$

where $n_{j}$ is the number of cells of type $j$ and $P_{j}: \mathbb{R}^{n} \rightarrow$ $\mathbb{R}^{n_{j}}$ is a projector such that $P_{j}(\boldsymbol{x})$ returns the values of $\boldsymbol{x}$ corresponding to the cells of type $j$.

This maximization is repeated until convergence of $\boldsymbol{\theta}_{t}$. The EM procedure does not guarantee to reach the global maximum of the likelihood function, since it can converge to a local maximum. However, in all the real and synthetic cases we studied, it gives excellent results and converges to the same point, independently of the initialization $\boldsymbol{\theta}_{0}$.

Once the previous step has converged, we have an estimation $\hat{\boldsymbol{\theta}}$ for the parameters of each cell type. Using this estimation in (10), we obtain $\hat{\boldsymbol{x}}_{\hat{\boldsymbol{\theta}}}$, an estimation for the TBs of each cell that maximizes the posterior distribution. This is the solution of the STAT method.

\section{Technical Aspects}

The geolocation errors of AMSR-2 products can reach several kilometers [8]. Given the high sensitivity of the object-based inverse methods to this kind of errors, a geocorrection step is performed before processing the images.

For a given displacement in the observations, the immensely overdetermined linear system (3) resulting from the discretization given by the original ground-based partition is solved with the least-squares (LSQR) method and the error $\| \boldsymbol{y}-$ $\boldsymbol{K} \hat{\boldsymbol{x}} \|$ is associated with the displacement. Our geocorrection algorithm assumes that this error is minimized when the given displacement compensates the original geolocation error. Concretely, using one variable for each object in the original ground-based partition, an LSQR method is run with the original geolocation and other eight geolocations consisting of the all possible combinations of $1 \mathrm{~km}$ displacements in a cardinal direction. The displacement leading to the least error is considered the new initial position and the procedure is iterated until convergence. Finally, the errors computed at the nine final positions are interpolated with a quadratic bivariate error function whose minimum is taken as the geocorrected position.

In this article, the antenna gain function is approximated by a bivariate Gaussian on the beam normal plane. Its shape is determined by the half-power beamwidth of the studied band. A basic kernel is computed projecting a generic antenna gain function onto a 1-km grid on the earth surface as done by Limaye et al. [15]. For the sake of finiteness, this basic kernel is clipped at $99 \%$ and renormalized. The basic kernel is then shifted and rotated to approximate any particular kernel, $G_{i}$. Given a retrieval grid, the cells are rasterized over the same $1-\mathrm{km}$ grid and the contributing fraction of the cells, $G_{i}^{j}$, are simply computed adding the values of the kernel $G_{i}$ over the pixels in the $j$ th cell.

\section{EVALUATION}

In this section, we use the proposed technique to grid real and synthetic AMSR-2 L1B measurements for different frequencies over the Pampa Deprimida, Buenos Aires, Argentina 


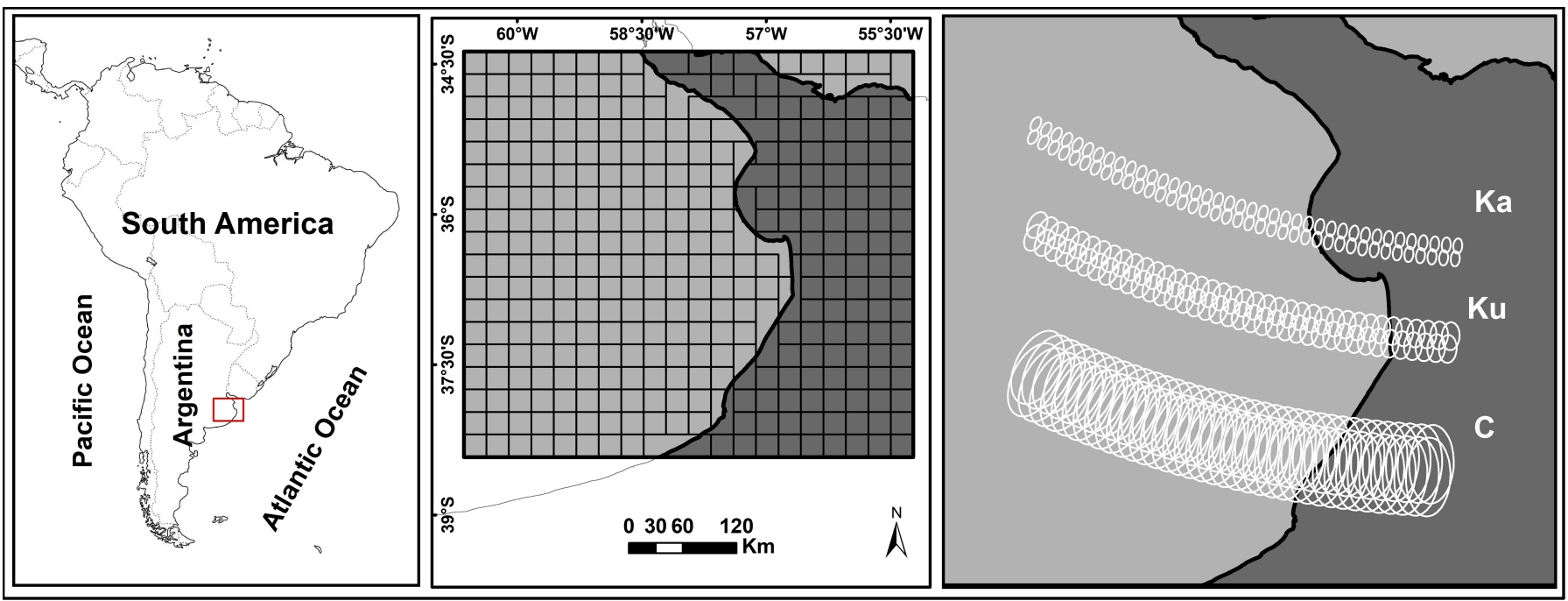

Fig. 2. Study region and some of the 3-dB footprints of the measurements used in the examples.

$\left(34^{\circ} 45^{\prime} 27^{\prime \prime} \mathrm{S}, 60^{\circ} 7^{\prime} 32^{\prime \prime} \mathrm{W}\right.$; $\left.38^{\circ} 7^{\prime} 45^{\prime \prime} \mathrm{S}, 55^{\circ} 41^{\prime} 56^{\prime \prime} \mathrm{W}\right)$. For this, we assume that the observation error has a normal distribution, with zero mean and a standard deviation of $1 \mathrm{~K}$, $\varepsilon \sim \mathcal{N}(0,1)$. The gridding was tested in five AMSR-2 bands with different half-power beam widths (C-band, $7.3 \mathrm{GHz}$, $35 \mathrm{~km} \times 61 \mathrm{~km}$; X-band, $10.7 \mathrm{GHz}, 24 \mathrm{~km} \times 42 \mathrm{~km}$; $\mathrm{K}_{\mathrm{u}}$-band, $18.7 \mathrm{GHz}, 13 \mathrm{~km} \times 22 \mathrm{~km}$; K-band, $23.8 \mathrm{GHz}$, $15 \mathrm{~km} \times 26 \mathrm{~km}$; and $\mathrm{K}_{\mathrm{a}}$-band, $36.5 \mathrm{GHz}, 7 \mathrm{~km} \times 12 \mathrm{~km}$ ) and in both horizontal (H-pol) and vertical polarizations for each band. The ground-based partition of the study region consists of two objects, labeled Land and Sea. Their limit is given by a cartographic digitalization of the coastline from high-resolution optic imagery. Fig. 2 shows the study area, the two objects of the ground-based partition, and the $3-\mathrm{dB}$ footprints of some sample measurements from each analyzed band.

Three retrieval grid sizes of $12.5,25$, and $50 \mathrm{~km}$ were tested for each band. The former is similar to the sampling interval of AMSR-2 measurements. The grid of $25 \mathrm{~km}$ is standard for many products from different sources, such as GLDAS, TMPA, ASCAT, and SMOS. The grids are regular, except in the coastal area where the ground-based partition intersects the cells. To keep the size of the coastal cells similar to that of the regular cells, when a resultant cell is smaller than half a regular cell, it is merged to the smallest adjacent one.

\section{A. Synthetic Observations}

The synthetic scenarios were created over the $12.5,25$ and $50-\mathrm{km}$ grids described in the previous paragraph. A TB value was assigned to each cell following two mechanisms. The first mechanism, which we call RAND, assigns a random value to each cell following a normal distribution whose parameters depend on the cell type. The TB for $i$ th cell is assigned as follows:

$$
\mathrm{TB}\left(C_{i}\right)= \begin{cases}270+10 z_{i}, & \text { if } l_{i}=\text { Land } \\ 180+5 z_{i}, & \text { if } l_{i}=\text { Sea }\end{cases}
$$

where $z_{i}$ is a random sample drawn from a standard normally distributed random variable.

The second mechanism, which we call TRIG, imposes a smoothness condition following a trigonometric pattern so that neighboring cells with the same type have similar values. This mechanism is similar to the one introduced by Bellerby et al. [14] for their simulations. We assign the truth value for each cell according to the following equation:

$\mathrm{TB}\left(C_{i}\right)= \begin{cases}270+20 \sin \left(\left(c_{i}^{y}-c_{i}^{x}\right) / 5\right), & \text { if } l_{i}=\text { Land } \\ 180+10 \sin \left(\left(c_{i}^{y}+c_{i}^{x}\right) / 5\right), & \text { if } l_{i}=\text { Sea }\end{cases}$

where $c_{i}^{y}$ and $c_{i}^{x}$ are the $i$ th cell geographical coordinates.

The constants involved in both mechanisms were selected so that the mean and standard deviation of the TB values corresponding to each surface cover type result similar to the corresponding values of the real scene processed in Section IV-E. Once the values $x_{i}=\mathrm{TB}\left(C_{i}\right)$ for each cell are computed (with either one mechanism), we generate a synthetic observation for each considered band. The simulated antenna TBs are given by

$$
y=\mathbf{K} x+\varepsilon
$$

where $\mathbf{K}$ is the kernel matrix corresponding to the simulated band and $\boldsymbol{\varepsilon}$ is a sample random error drawn from the error distribution described above.

For each combination of retrieval grids $(12,25$, and $50 \mathrm{~km})$, bands $\left(\mathrm{K}_{\mathrm{a}}, \mathrm{K}, \mathrm{K}_{\mathrm{u}}, \mathrm{X}\right.$, and $\left.\mathrm{C}\right)$, and generation mechanisms (RAND and TRIG), we obtain a synthetic image and a corresponding synthetic observation.

To evaluate the methods, we compared the estimated values, $\hat{\boldsymbol{x}}$, with the synthetic values, $\boldsymbol{x}$, computing both the RMSE, defined as

$$
\mathrm{RMSE}=\sqrt{\frac{1}{n} \sum_{i=1}^{n}\left(x_{i}-\hat{x}_{i}\right)^{2}}
$$

and the Pearson correlation coefficient, $\rho(\boldsymbol{x}, \hat{\boldsymbol{x}})$. 
TABLE I

Mean and Standard Deviation of the Obtained RMSE and the Mean Correlation Coefficient $(\rho)$ For the 100 Replications of the (Top) RAND AND (BOTTOM) TRIG S YNTHETIC EXPERIMENTS, SOlved OVER (LEFT) 12.5-km AND (RIGHT) 25-km GRIDS, CONSIDERING THE STAT, BG, AND LSQR MethodS AND The Five Bands $\left(\mathrm{K}_{\mathrm{A}}, \mathrm{K}_{\mathrm{U}}, \mathrm{K}, \mathrm{X}\right.$, AND C)

\begin{tabular}{|c|c|c|c|c|c|c|c|c|c|c|c|c|}
\hline \multicolumn{2}{|c|}{ STAT $12.5 \mathrm{~km}$} & \multicolumn{2}{|c|}{ BG $12.5 \mathrm{~km}$} & \multicolumn{2}{|c|}{ LSQR $12.5 \mathrm{~km}$} & \multirow[t]{2}{*}{ Band } & \multicolumn{2}{|c|}{ STAT $25 \mathrm{~km}$} & \multicolumn{2}{|c|}{ BG $25 \mathrm{~km}$} & \multicolumn{2}{|c|}{ LSQR $25 \mathrm{~km}$} \\
\hline RMSE & $\rho$ & RMSE & $\rho$ & RMSE & $\rho$ & & RMSE & $\rho$ & RMSE & $\rho$ & RMSE & $\rho$ \\
\hline \multicolumn{13}{|c|}{ RAND experiments } \\
\hline $1.38(0.04)$ & 1.00 & $5.05(0.18)$ & 0.99 & $1.42(0.04)$ & 1.00 & $\mathrm{~K}_{\mathrm{a}}$ & $0.46(0.02)$ & 1.00 & $0.94(0.05)$ & 1.00 & $0.46(0.02)$ & 1.00 \\
\hline $4.16(0.17)$ & 1.00 & $7.95(0.23)$ & 0.98 & $7.81(0.48)$ & 0.98 & $\mathrm{~K}_{\mathrm{u}}$ & $0.65(0.03)$ & 1.00 & $2.67(0.14)$ & 1.00 & $0.65(0.03)$ & 1.00 \\
\hline $5.07(0.22)$ & 0.99 & $8.66(0.26)$ & 0.98 & $18.9(1.41)$ & 0.91 & $\mathrm{~K}$ & $0.75(0.04)$ & 1.00 & $3.55(0.18)$ & 1.00 & $0.76(0.04)$ & 1.00 \\
\hline $7.25(0.24)$ & 0.99 & $11.57(0.31)$ & 0.96 & $689.5(423)$ & 0.06 & $\mathrm{X}$ & $2.20(0.19)$ & 1.00 & $7.39(0.34)$ & 0.99 & $2.65(0.30)$ & 1.00 \\
\hline $8.11(0.22)$ & 0.98 & $13.66(0.33)$ & 0.95 & $1047(61)$ & 0.04 & $\mathrm{C}$ & $4.89(0.24)$ & 0.99 & $10.51(0.5)$ & 0.97 & $43.89(7.6)$ & 0.70 \\
\hline \multicolumn{13}{|c|}{ TRIG experiments } \\
\hline $1.35(0.03)$ & 1.00 & $3.11(0.02)$ & 1.00 & $1.41(0.04)$ & 1.00 & $\mathrm{~K}_{\mathrm{a}}$ & $0.46(0.02)$ & 1.00 & $0.85(0.04)$ & 1.00 & $0.46(0.02)$ & 1.00 \\
\hline $3.19(0.11)$ & 1.00 & $4.98(0.03)$ & 0.99 & $7.79(0.54)$ & 0.98 & $\mathrm{~K}_{\mathrm{u}}$ & $0.64(0.03)$ & 1.00 & $2.18(0.07)$ & 1.00 & $0.65(0.03)$ & 1.00 \\
\hline $3.40(0.13)$ & 1.00 & $5.50(0.04)$ & 0.99 & $19.08(1.50)$ & 0.91 & $\mathrm{~K}$ & $0.75(0.04)$ & 1.00 & $2.74(0.08)$ & 1.00 & $0.76(0.04)$ & 1.00 \\
\hline $3.82(0.16)$ & 1.00 & $8.31(0.04)$ & 0.98 & 694.5 (43) & 0.06 & $\mathrm{X}$ & $1.94(0.16)$ & 1.00 & $5.39(0.11)$ & 0.99 & $2.66(0.28)$ & 1.00 \\
\hline $3.73(0.21)$ & 1.00 & $10.66(0.03)$ & 0.97 & $1053(61)$ & 0.04 & $\mathrm{C}$ & $2.90(0.23)$ & 1.00 & $8.02(0.15)$ & 0.98 & $40.19(6.7)$ & 0.73 \\
\hline
\end{tabular}

\section{B. Methods Comparison}

The STAT, BG, and LSQR methods were used to obtain estimates, $\hat{\boldsymbol{x}}$, for the TB of each cell, for each set of synthetic observations. Some graphical results are shown in Fig. 3.

For each grid size (12.5 and $25 \mathrm{~km}$.), $10 \mathrm{TRIG}$ and 10 RAND synthetic images were created. For each of these images, 10 noisy AMSR-2 observations were simulated. The mean and standard deviation of the obtained RMSE and the mean correlation values for these experiments are given in Table I.

From the results of the experiments, we observe that the LSQR solution presents typically wild oscillations since the measurement errors are amplified. As can be appreciated in Fig. 3 and Table I, the noise amplification can be controlled at the expense of a loss in the information content of the solution, forcing a gross observation oversampling, i.e., using large cells compared with the sampling resolution (cf. [15]). In these cases, the LSQR method gives excellent results.

The BG method is more robust and gives reasonable results in homogeneous areas. However, it introduces an undesirable blurring effect in regions with high TB contrast. The correlation coefficient shows that the BG solution is highly correlated with the original image. However, the RMSE shows an unadmissible level of disagreement for all the images solved over the $12.5-\mathrm{km}$ retrieval grid and most of the images bands solved over the $25-\mathrm{km}$ retrieval grid.

In the solutions given by the STAT method, it is remarkable the removal of the coastal blurring effect. Also, higher correlation coefficients and lower RMSE are obtained, compared with both benchmark methods.

In the synthetic experiments solved with the STAT method, we observe that the estimated mean temperature of each cell type is accurate but the estimated standard deviation suffers a slight underestimation. This may happen since the maximum likelihood estimator is generally consistent but not always unbiased.
TABLE II

MEAN RMSE FOR BORDER AND INTERIOR CELLS FOR THE 100 REPLICATIONS OF THE TRIG SYNTHETIC EXPERIMENT, SOLVED OVER THE 25-km GRID, CONSIDERING THE STAT, BG, AND LSQR METHODS AND THE Five BANDS $\left(\mathrm{K}_{\mathrm{A}}, \mathrm{K}_{\mathrm{U}}, \mathrm{K}, \mathrm{X}\right.$, AND C)

\begin{tabular}{|c|cc|cc|cc|}
\hline Band & \multicolumn{2}{|c|}{ STAT $25 \mathrm{~km}$} & \multicolumn{2}{c|}{ BG $25 \mathrm{~km}$} & \multicolumn{2}{c|}{ LSQR $25 \mathrm{~km}$} \\
& Border & Int. & Border & Int. & Border & Int. \\
\hline $\mathrm{K}_{\mathrm{a}}$ & 0.41 & 0.46 & 1.75 & 0.67 & 0.42 & 0.46 \\
$\mathrm{~K}_{\mathrm{u}}$ & 0.55 & 0.65 & 6.28 & 1.24 & 0.56 & 0.65 \\
$\mathrm{~K}$ & 0.58 & 0.77 & 8.10 & 1.35 & 0.59 & 0.78 \\
$\mathrm{X}$ & 1.42 & 1.97 & 16.66 & 2.16 & 1.89 & 2.63 \\
$\mathrm{C}$ & 2.51 & 2.93 & 23.55 & 3.87 & 14.92 & 41.03 \\
\hline
\end{tabular}

The retrieval procedure for a single image required less than $1 \mathrm{~s}$ running on a standard desktop computer with an Intel Quad-Core i7 processor and 16 GB of RAM.

For simplicity, our prior assumes the independence of the state variables. However, TBs of adjacent cells are typically correlated. The results of the RAND and TRIG synthetic experiments (see Table I) suggest that when regional tendencies in the TB field exist, the STAT method improves its precision.

To further analyze the differences among the three retrieval methods, in Table II, we present the mean RMSE for the 100 replications of the TRIG experiment over the $25-\mathrm{km}$ grid, discriminated for interior and coastal cells. The blurring effect caused by the BG method induces a much higher RMSE in the coastal cells as compared to the interior cells. The STAT method outperforms both BG and LSQR methods for both interior and coastal cells. It is interesting to observe that the RMSE of STAT method is lower for the coastal cells. This is a consequence of the merging algorithm for irregular cells, in the case of $25-\mathrm{km}$ grid, which leads to coastal cells that are $30 \%$ larger than regular cells (on average), increasing the information available for the retrieval of each coastal cell TB value. A similar phenomenon can be observed for the 


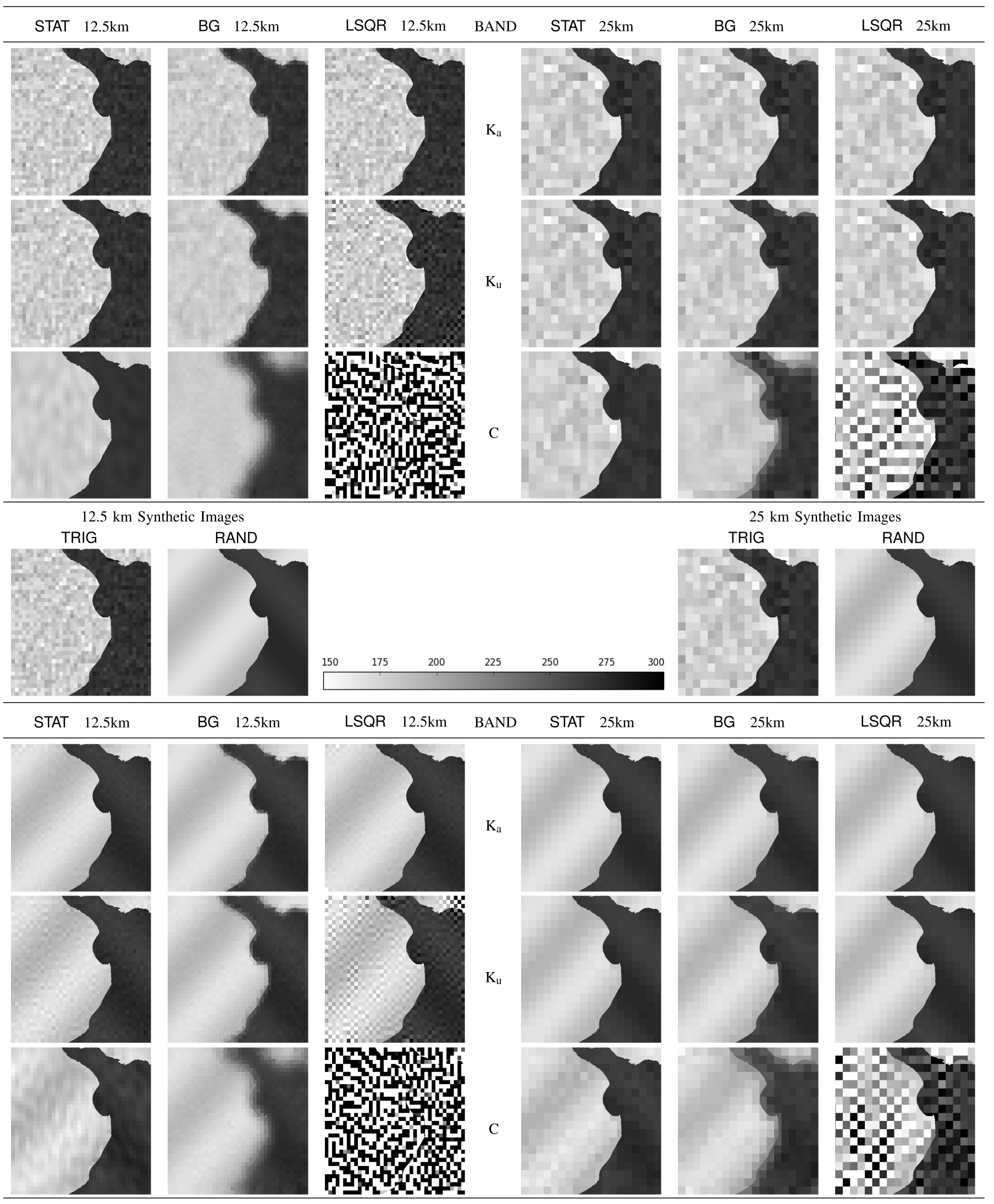

Fig. 3. Results of (Top)TRIG and (Bottom) RAND synthetic experiments for the three methods (STAT, BG, and LSQR) and three bands ( $K_{a}$, $K_{u}$, and C) solved over (Left) $12.5-\mathrm{km}$ and (Right) 25-km grids. (Middle) Reference synthetic images. 
LSQR method, where larger coastal cells lead to smaller noise amplification.

Each band has an adequate retrieval grid size, depending on the application. Since different bands have the same spatial sampling frequency, this fact is not related to an algebraic dimensionality problem and it seems to correspond to the information content of the observational data, which is degraded as the size of the footprint increases. For instance, the kernel matrices corresponding to the synthetic experiments with resolutions $12.5 \mathrm{~km}$ and $25 \mathrm{~km}$ have ranks 1465 and 235, respectively, independent of the processed band. However, the degrees of freedom for signal [19] which describe the number of useful independent quantities contained in the observations and is a measure of information, changes with the band. For $12.5-\mathrm{km}$ resolution, the observations have 802.2, 355.3, 282.1, 115.0, and 51.2 degrees of freedom for signal for bands $\mathrm{K}_{\mathrm{a}}, \mathrm{K}_{\mathrm{u}}, \mathrm{K}, \mathrm{X}$, and $\mathrm{C}$, respectively. For $25-\mathrm{km}$ resolution, the degrees of freedom for signal for the analyzed bands are, respectively, 228.2, 220.1, 215.1, 158.9, and 89.7.

If we assume an emission model analogous to RAND or TRIG and admit an expected RMSE of $2{ }^{\circ} \mathrm{K}$ in the TB retrieval process, then the $\mathrm{K}_{\mathrm{a}}$-band can be retrieved in the $12.5-\mathrm{km}$ grid, whereas the $\mathrm{K}_{\mathrm{u}^{-}}$and $\mathrm{K}$-bands should be retrieved in the $25-\mathrm{km}$ grid (see Table I). The retrieval $\mathrm{X}$ - and C-bands in the $50-\mathrm{km}$ grid would produce RMSE errors bounded by 0.30 and 0.52 , respectively. These resolutions will depend on the spectral characteristics of the objects present in the scene and on the microwave radiometer. The values presented here are valid in this particular context.

\section{Influence of Instrument Error on the Solutions}

To study the robustness of the different proposed methods with respect to the instrument noise level, we ran synthetic experiments varying the observational error standard deviation, $\sigma_{\text {obs. }}$. We present the results of these experiments for the K-band, solved over a $25-\mathrm{km}$ resolution grid. The results are analogous for the different grid resolution levels and different bands discussed in this paper.

Fig. 4 shows the RMSE for the STAT, LSQR, and BG solution with different noise levels. The RMSE shown is the average of 10 replications. It can be seen that while the LSQR method induces an error that is almost linear in the observational error, both BG and STAT methods are able to reduce the influence of the observational error on the RMSE.

The LSQR method has an excellent performance with low instrumental noise and fine resolution bands. However, it breaks down as the noise level increases or the observational resolution decreases (see Table I). The BG method is robust in the sense that its RMSE is bounded even for high noise levels, but it underperforms LSQR for low noise levels. In all tested scenarios, the STAT method outperformed both BG and LSQR. It has the advantage of both the robustness of $B G$ and the good performance of LSQR for low noise level and fine resolution bands.

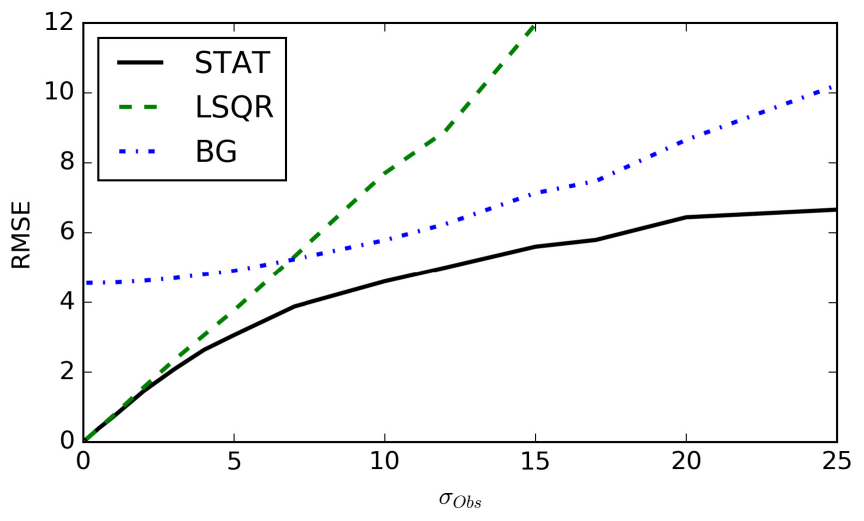

Fig. 4. RMSE for the solutions computed with STAT, LSQR, and BG methods for different noise levels. TRIG synthetic image with K-band observations solved over the $25-\mathrm{km}$ grid.

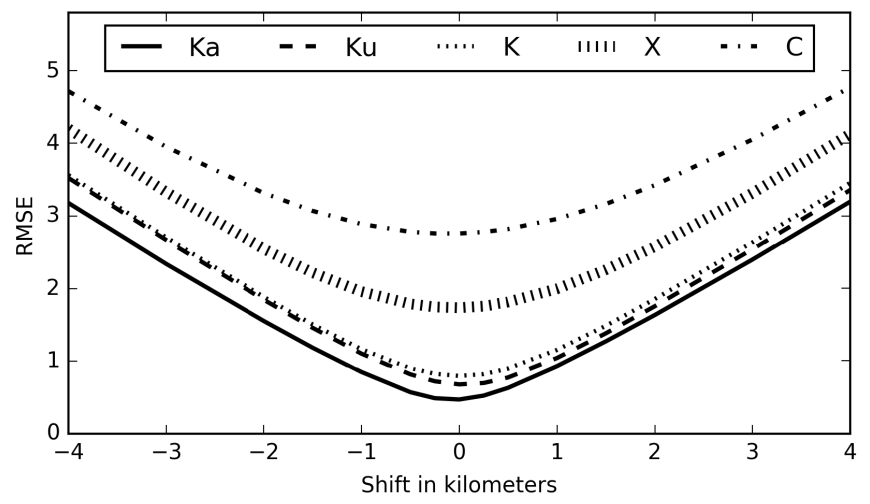

Fig. 5. Result of geolocation error on the image reconstruction RMSE for the $25-\mathrm{km}$ grid and TRIG synthetic image solved with the STAT method.

\section{Geolocation Error Analysis for STAT}

Since the STAT reconstruction method assumes different Gaussian models for the different objects in the scene, a uniform geolocation error in the observations (or in the underlying ground-based partition) does not translate into a uniform shift of the reconstructed TB values; it has impact on the parameters for each cell-type model, affecting the complete reconstruction procedure.

To study the error induced on the reconstruction of the cell TBs by geolocation errors, the following analysis was performed for the STAT method. Geolocation errors were simulated by shifting a fixed distance the longitude of the synthetic observations for different bands and solved over different grids. In Fig. 5, we plotted the RMSE obtained when using the STAT inverse method over the $25-\mathrm{km}$ retrieval grid with shifted observations.

When no geolocation error is introduced, the RMSE values are the ones given by Table I. As the geolocation error increases, the RMSE also increases at a rate lower than one degree per kilometer. The experiments suggest that the curve slope is independent of the band.

This analysis shows that an imperfect ground-based image partition increases the retrieved solution RMSE. We emphasize that, as expected, this method works better when the objects' 


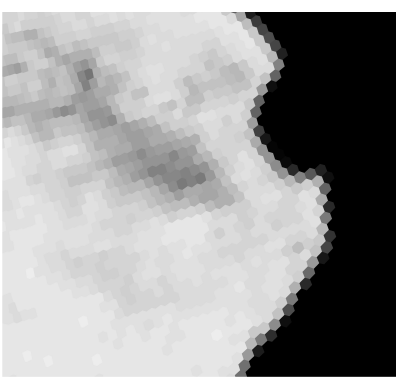

(a)

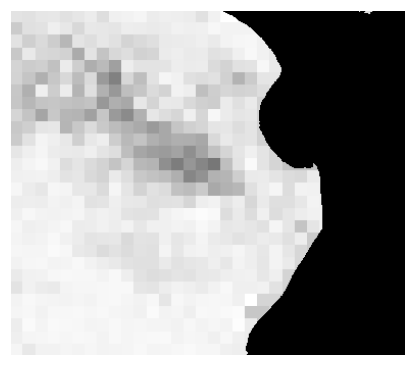

(c)

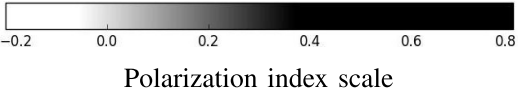

Fig. 6. Polarization indices computed for different bands and methods from real AMSR-2 data from June 2, 2014. (a) Polarization index from raw data, $\mathrm{K}_{\mathrm{a}}$-band, nearest neighbor. (b) Polarization index from raw data, X-band, nearest neighbor. (c) Polarization index from STAT solution for $\mathrm{K}_{\mathrm{a}}$-band at $12.5-\mathrm{km}$ resolution grid. (d) Polarization index from STAT solution for X-band at $25-\mathrm{km}$ resolution grid.

borders are correctly defined and the observations are correctly geolocated.

\section{E. Real Observations}

The statistical inverse method is applied to real AMSR-2 observations (descending orbit 220) acquired on June 2, 2014 at the Pampa Deprimida.

Pampa Deprimida has a very low regional slope (lower to the east) with numerous depressions caused by eolic deflation processes during the quaternary dry periods. Water accumulates in these depressions during rains and is then lost by evaporation and infiltration. Thus, the drainage network is endorreic or arreic most of the time and is only integrated during important flooding events. Vegetation is mainly herbaceous, ranging from grasslands to hydrophytes. This region is highly channelized and the main activities include agriculture and cattle farming, according to the flooding frequency.

The selected scene includes a flooded area that crosses from the NW corner to the center of the study region, corresponding to a moderate inundation.

Fig. 6 compares the polarization index $\left(\mathrm{PI}=\left(\left(\mathrm{TB}_{v}-\mathrm{TB}_{h}\right) /\right.\right.$ $\left.\left(\mathrm{TB}_{v}+\mathrm{TB}_{h}\right)\right)$ ) computed from both the raw data and the STAT solution for $\mathrm{K}_{\mathrm{a}}$ - and $\mathrm{X}$-bands.

A comparison of Fig. 6(a) and (c) shows the improvements obtained by the STAT method over the original raw data for the

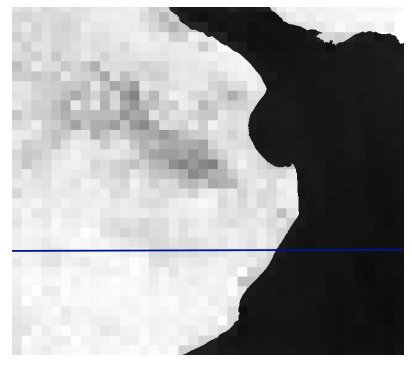

(a)

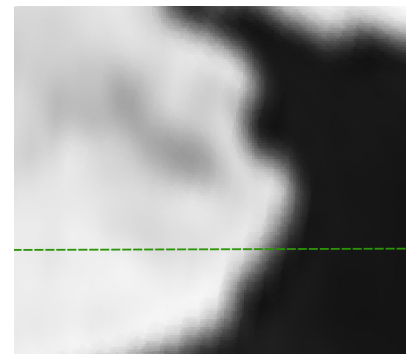

(b)

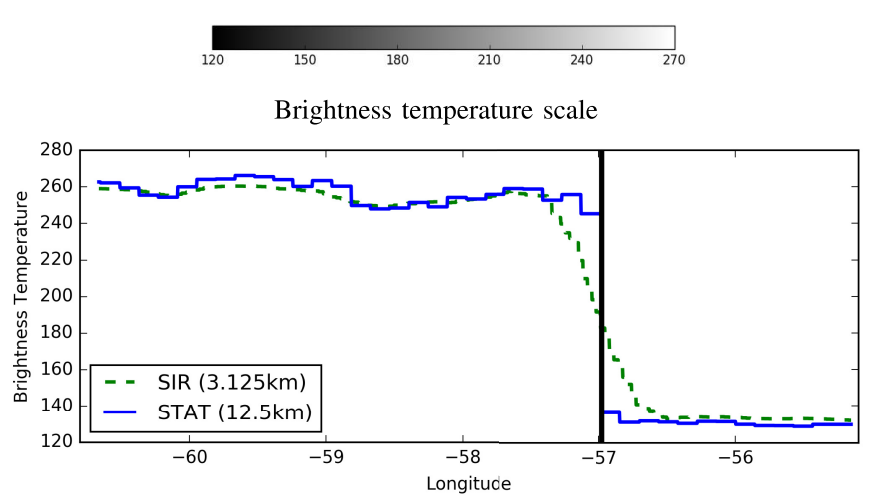

(c)

Fig. 7. Comparison of the STAT method $(12.5-\mathrm{km}$ resolution) with the enhanced resolution NSIDC product $(3.125-\mathrm{km}$ resolution). Data from June 2, 2014, ascending orbits, H-pol. The values of both images were evaluated over the same transect and the result plotted in (c). Both products have a good agreement far from the coast. A blurring effect can be perceived in the NSIDC product. (a) AMSR-2 $\mathrm{K}_{\mathrm{a}}$-band $\mathrm{H}$-pol data gridded with the STAT method at $12.5 \mathrm{~km}$. (b) SSMIS $\mathrm{K}_{\mathrm{a}}$-band $\mathrm{H}$-pol data gridded with the SIR method at $3.125 \mathrm{~km}$. (c) Transect showing TB values from above images at latitude $37.27^{\circ}$. Black vertical line: location of the coastline.

$\mathrm{K}_{\mathrm{a}}$-band. The first remarkable difference is the sharp coastline visible in Fig. 6(c) as opposed to the fuzzy border visible in Fig. 6(a). The STAT method leads to coastal pixels with pure land or sea TB values, in contrast to the original mixed values. This fact is of utmost importance for coastal retrieval applications. At the same time, we can observe that the STAT method is able to deconvolve the measurements reobtaining the extreme values for features present over the land area, even when their exact boundaries are not designed in the retrieval grid. This is the case for the shallow lakes present in the SE portion of the scene and the flooded area in the NW portion of the study region. Since the X-band footprint is larger, the X-band observations contain less information and its retrieval is more difficult. We perform it over the $25-\mathrm{km}$ grid. A comparison of Fig. 6(b) and (d) shows that the above mentioned is not dependent on the grid resolution, since both the sharp coastline and the delineation of the main features inside the land area also appear in the $25-\mathrm{km}$ grid. We also observe that Fig. 6(d) is smoother than Fig. 6(b). We interpret this as a consequence of the blurring effect induced by large footprints. The STAT method is able to deconvolve the extreme values present in the scene when solved over an adequate resolution grid. 
In Fig. 7, we compare the results obtained by the STAT method with the enhanced-resolution-gridded product offered by the National Snow and Ice Data center (NSIDC) (data set ID: NSIDC-0630, see [22]). Fig. 7(a) corresponds to the AMSR-2 data mentioned above, retrieved with the STAT method over a 12.5-km grid, while Fig. 7(b) corresponds to Special Sensor Microwave Image/Sounder (SSMIS) data resampled using the SIR algorithm at $3.125 \mathrm{~km}$, as proposed in [23]. Both data sets were acquired on June 2, 2014, with ascending orbits and H-pol.

A simple visual comparison of Fig. 7(a) and (b) shows, again in this case, that the STAT method leads to coastal pixels with pure land or sea TB values, in contrast to the SIR method that has mixed values.

To further analyze the differences, the values of both images were evaluated over the same transect and the result is plotted in Fig. 7(c). We observe that the solid line (STAT) presents an abrupt change of TB in the coastal area, while the dashed line (SIR) presents a smooth transition. The figure also shows that both products have a good agreement far from the coast.

\section{CONCLUSION}

We introduced a statistical inverse method for gridding passive microwave data with mixed measurements. The method uses geospatial information about the spectral objects present in the scene to allow the computation of accurate TBs near their edges, avoiding the introduction of artifacts and an excessive noise amplification. It uses a priori information consisting on the assumption that there are given different cell types and that the TB corresponding to the cells of each type share a common probabilistic distribution. While standard least-squares regression can be useful for fine-resolution bands where there is enough information for a proper retrieval, its noise amplification rapidly renders it inadequate for coarser bands retrieval. On the other hand, BG method is more robust but it proved to be quite inefficient regarding its capability to solve the problem without the introduction of a blurring effect near the objects' borders. Our method combines the advantage of both methodologies: it includes the efficiency of least squares and the robustness of BG, surpassing both methods in all scenarios.

In that sense, we have established that the incorporation of a priori information about the different cell types can be beneficial for the inverse problem resolution. This information is usually available but requires an adequate statistical model to be properly incorporated.

The STAT method can be applied in a variety of situations, including coastal zones, agricultural fields, floodplains, and to all regions with an abrupt change in their ecogeomorphologic features. However, the actual boundaries of the different spectral objects considered in the scene need to be precisely known. The method may also be used in situations where there is an object whose limits are not precisely known, but in that case, the object should not be distinguished in the prior, with the consequent loss in the retrieval performance. For instance, if a lake considered in the prior suffers an important increase on its surface but its border is not updated accordingly, the retrieval of its TB might lead to inaccurate values. On the other hand, if the lake is not considered, the retrieval will lead to blurred temperatures (which may be preferable). The ideal situation is, of course, to know the precise updated boundary of the lake.

The STAT method is general and can be applied to any sensor whose antenna gain function and incidence angle are known. We applied it to AMSR-2 data to illustrate its application, but it may be used to grid SMOS, SMAP, SSM/I, SSMIS, or AMSR-E measurements.

Since our technique can be applied to grid measurements from different frequencies on the same grid, it offers a solution to the problem of simultaneously analyzing measurements acquired at different frequencies by antennas with noncoincident patterns as is required to compute multifrequency indices.

Ideally, heterogeneity between the cells should be greater than within each cell. In many cases, this requires the inclusion of spectral objects smaller than the retrieval grid resolution in the ground-based segmentation. This can be done, in principle, especially if these small objects form a pattern of multiple segments with similar spectral characteristics. Also, the large sparse objects such as rivers may be included in the partition. However, the current geolocation errors hinder an adequate resolution of these situations.

The statistical method cannot be directly applied at large scales. For instance, to grid a complete AMSR-2 orbit comprising almost 500000 observations, the use of sparse linear algebra methods would be required.

\section{ACKNOWLEDGMENT}

The authors would like to thank the anonymous reviewers for their valuable comments and suggestions to improve the paper. They would also like to thank M. Cerdeiro for all her helpful comments and suggestions.

\section{REFERENCES}

[1] F. T. Ulaby, R. K. Moore, and A. K. Fung, Microwave Remote Sensing: Active and Passive, vols. 1-3. Norwood, MA, USA: Artech House, 1981.

[2] B. J. Choudhury, "Monitoring global land surface using Nimbus-7 $37 \mathrm{GHz}$ data theory and examples" Int. J. Remote Sens., vol. 10, no. 10, pp. 1579-1605, 1989

[3] E. G. Njoku, T. J. Jackson, V. Lakshmi, T. K. Chan, and S. V. Nghiem, "Soil moisture retrieval from AMSR-E," IEEE Trans. Geosci. Remote Sens., vol. 41, no. 2, pp. 215-229, Feb. 2003.

[4] L. A. Jones, J. S. Kimball, K. C. McDonald, S. T. K. Chan, E. G. Njoku, and W. C. Oechel, "Satellite microwave remote sensing of boreal and arctic soil temperatures from AMSR-E," IEEE Trans. Geosci. Remote Sens., vol. 45, no. 7, pp. 2004-2018, Jul. 2007.

[5] L. Zhang, T. Zhao, L. Jiang, and S. Zhao, "Estimate of phase transition water content in freeze-thaw process using microwave radiometer," IEEE Trans. Geosci. Remote Sens., vol. 48, no. 12, pp. 4248-4255, Dec. 2010.

[6] S. J. Sippel, S. K. Hamilton, J. M. Melack, and B. J. Choudhury, "Determination of inundation area in the Amazon River floodplain using the SMMR $37 \mathrm{GHz}$ polarization difference," Remote Sens. Environ., vol. 48, no. 1, pp. 70-76, 1994.

[7] Q. Min, B. Lin, and R. Li, "Remote sensing vegetation hydrological states using passive microwave measurements," IEEE J. Sel. Topics Appl. Earth Observ. Remote Sens., vol. 3, no. 1, pp. 124-131, Mar. 2010.

[8] Descriptions of GCOM-W1 AMSR2 Level $1 R$ and Level 2 Algorithms. Japan Aerosp. Explor. Agency, Earth Observ. Res. Center, Tokyo, Japan, 2013. 
[9] V. Barraza et al., "Monitoring vegetation moisture using passive microwave and optical indices in the dry Chaco forest, argentina," IEEE J. Sel. Topics Appl. Earth Observat. Remote Sens., vol. 7, no. 2, pp. 421-430, Feb. 2014.

[10] G. Backus and F. Gilbert, "Uniqueness in the inversion of inaccurate gross earth data," Philos. Trans. Roy. Soc. London A, Math. Phys. Sci., vol. 266, no. 1173, pp. 123-192, Mar. 1970.

[11] A. Stogryn, "Estimates of brightness temperatures from scanning radiometer data," IEEE Trans. Antennas Propag., vol. AP-26, no. 5, pp. 720-726, Sep. 1978.

[12] G. A. Poe, "Optimum interpolation of imaging microwave radiometer data," IEEE Trans. Geosci. Remote Sens., vol. 28, no. 5, pp. 800-810, Sep. 1990.

[13] M. R. Farrar and E. A. Smith, "Spatial resolution enhancement of terrestrial features using deconvolved SSM/I microwave brightness temperatures," IEEE Trans. Geosci. Remote Sens., vol. 30, no. 2, pp. 349-355, Mar. 1992.

[14] T. Bellerby et al., "Retrieval of land and sea brightness temperatures from mixed coastal pixels in passive microwave data," IEEE Trans. Geosci. Remote Sens., vol. 36, no. 6, pp. 1844-1851, Nov. 1998.

[15] A. S. Limaye, W. L. Crosson, C. A. Laymon, and E. G. Njoku, "Land cover-based optimal deconvolution of PALS L-band microwave brightness temperatures," Remote Sens. Environ., vol. 92, no. 4, pp. 497-506, 2004.

[16] T. Blaschke, "Object based image analysis for remote sensing," ISPRS J. Photogram. Remote Sens., vol. 65, no. 1, pp. 2-16, Jan. 2010.

[17] T. Blaschke et al., "Geographic object-based image analysis-Towards a new paradigm," ISPRS J. Photogramm. Remote Sens., vol. 87, pp. 180-191, Jan. 2014.

[18] W. G. Rees, Physical Principles of Remote Sensing. Cambridge, U.K.: Cambridge Univ. Press, 2013

[19] C. D. Rodgers, Inverse Methods for Atmospheric Sounding: Theory and Practice, vol. 2. Singapore: World scientific, 2000.

[20] K. P. Murphy, Machine Learning-A Probabilistic Perspective. Cambridge, MA, USA: MIT Press, 2012.

[21] A. P. Dempster, N. M. Laird, and D. B. Rubin, "Maximum likelihood from incomplete data via the EM algorithm," J. Roy. Statist. Soc., B (Methodol.), vol. 39, no. 1, pp. 1-38, 1977.

[22] M. Brodzik, D. Long, M. Hardman, A. Paget, and R. Armstrong, MEaSUREs Calibrated Enhanced-Resolution Passive Microwave Daily EASE-Grid 2.0 Brightness Temperature ESDR, Version 1. NASA Nat. Snow Ice Data Center Distrib. Act. Arch. Center, Washington, DC, USA, 2018.

[23] D. G. Long and D. L. Daum, "Spatial resolution enhancement of SSM/I data," IEEE Trans. Geosci. Remote Sens., vol. 36, no. 2, pp. 407-417, Mar. 1998.

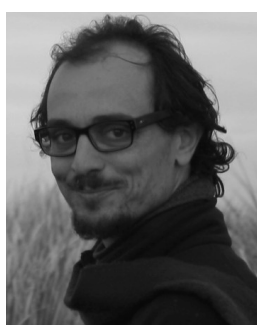

Rafael Grimson received the Licenciatura degree in mathematics from the University of Buenos Aires, Buenos Aires, Argentina, in 2001, the master's degree in foundations of computer science from École Polytechnique, Paris, France, in 2005, and the Ph.D. degree from Hasselt University, Hasselt, Belgium, in 2010.

$\mathrm{He}$ is currently a CONICET Researcher at the Remote Sensing Laboratory, Environmental Engineering Research Institute, Universidad Nacional de San Martín (LETyE, 3iA, UNSAM), San Martín, Argentina. His research interests include remote sensing, machine learning, and hydrologic modeling.

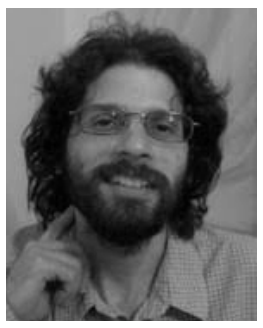

Juan Lucas Bali received the Licenciatura degrees in computer science and mathematics and the Ph.D. degree in mathematical statistics from the University of Buenos Aires, Buenos Aires, Argentina, in 2004, 2008, and 2012, respectively.

$\mathrm{He}$ is currently a CONICET Researcher at the Instituto de Investigaciones Científicas y Tecnicas para la Defensa, Villa Martelli, Argentina. His research interests include the mathematical modeling of meteorological phenomena and the use of inverse methods for remote sensing with Lidar technology.

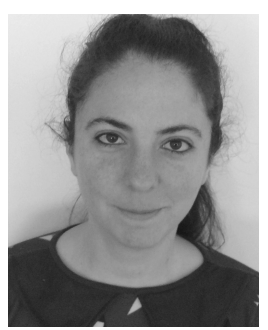

Mariela Rajngewerc received the Licenciatura degree in mathematics from the University of Buenos Aires, Buenos Aires, Argentina, in 2016. She is currently pursuing the Ph.D. degree at $3 \mathrm{iA}$, Universidad Nacional de San Martín, San Martín, Argentina, under the supervision of R. Grimson, with a focus on the design of algorithms for wetlands studies based on SAR data.

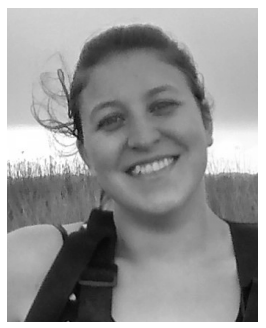

Laura San Martín received the Licenciatura degree in geology from the University of Buenos Aires, Buenos Aires, Argentina, in 2012. She is currently pursuing the $\mathrm{Ph} . \mathrm{D}$. degree at $\mathrm{BA}$, Universidad Nacional de San Martín, San Martín, Argentina.

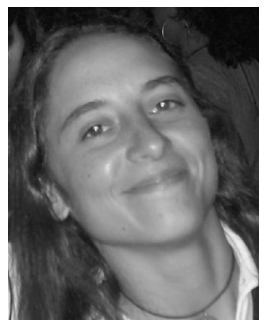

Mercedes Salvia (M'08) received the Licenciatura degree in biology and the Ph.D. degree from the University of Buenos Aires, Buenos Aires, Argentina, in 2004 and 2010, respectively.

She is currently a CONICET Researcher at the Remote Sensing Laboratory, Astronomy and Space Physics Institute (IAFE, UBA-CONICET), Buenos Aires. Her research interests include the analysis temporal series of optical and microwave satellite images to study forests, climate, and wetland dynamics. 